\title{
Cumulative Activation of Voltage-Dependent KVS-1 Potassium Channels
}

\author{
Patricio Rojas, ${ }^{1}$ Jonathan Garst-Orozco, ${ }^{1}$ Beravan Baban, ${ }^{1}$ \\ Jose Antonio de Santiago-Castillo, ${ }^{3}$ Manuel Covarrubias, ${ }^{3}$ and Lawrence Salkoff ${ }^{1,2}$ \\ Departments of ${ }^{1}$ Anatomy and Neurobiology and ${ }^{2}$ Genetics, Washington University School of Medicine, St. Louis, Missouri 63110, and ${ }^{3}$ Department of \\ Pathology, Anatomy, and Cell Biology, Jefferson Medical College of Thomas Jefferson University, Philadelphia, Pennsylvania 19107
}

In this study, we reveal the existence of a novel use-dependent phenomenon in potassium channels, which we refer to as cumulative activation (CA). CA consists of an increase in current amplitude in response to repetitive depolarizing step pulses to the same potential. CA persists for up to $20 \mathrm{~s}$ and is similar to a phenomenon called "voltage-dependent facilitation" observed in some calcium channels. The KVS-1 K ${ }^{+}$channel, which exhibits CA, is a rapidly activating and inactivating voltage-dependent potassium channel expressed in chemosensory and other neurons of Caenorhabditis elegans. It is unusual in being most closely related to the Shab (Kv2) family of potassium channels, which typically behave like delayed rectifier $\mathrm{K}^{+}$channels in other species. The magnitude of CA depends on the frequency, voltage, and duration of the depolarizing step pulse. CA also radically changes the activation and inactivation kinetics of the channel, suggesting that the channel may undergo a physical modification in a use-dependent manner; thus, a model that closely simulates the behavior of the channel postulates the existence of two populations of channels, unmodified and modified. Use-dependent changes in the behavior of potassium channels, such as CA observed in KVS-1, could be involved in functional mechanisms of cellular plasticity such as synaptic depression that represent the cellular basis of learning and memory.

Key words: potassium channels; facilitation; inactivation; use dependency; plasticity; physiology

\section{Introduction}

Voltage-dependent potassium channels are key players in tuning electrical signals in excitable cells. Modifications in potassium channel activity by accessory subunits, secondary messenger cascades, splice variants, and other modifiers can account for changes in electrical responses. In 1979, Aldrich et al. described a peculiar use-dependent behavior, which they termed "cumulative inactivation," of a potassium current in molluscan neurons. In their observations, they described a progressive frequencydependent decline in outward current amplitude in response to repetitive depolarization. When two depolarizing pulses are repeated at frequencies near $1 \mathrm{~Hz}$, the peak outward current during the second pulse is less than the current at the end of the preceding pulse. This pattern of outward current gives the impression that the availability of outward current channels continues to decline during the repolarized interval. This feature of cumulative inactivation was subsequently seen in many species and cell types. We now report the reverse phenomenon, which we term "cumulative activation" (CA). In the KVS-1 channel, cloned from Caenorhabditis elegans (Bianchi et al., 2003), we observe a

Received Aug. 21, 2007; revised Nov. 29, 2007; accepted Dec. 1, 2007

This work was supported by National Institute of Health Grants R24 RR017342-01 and R01 GM067154-01A1, and M.C. and J.D.S.C. were supported by R01 NS032337-13 (M.C.). We thank Dr. Federico Sesti for kindly providing the KVS-1 CDNA, Veronica West and Julia Kuhn for Xenopus 0ocyte isolation, and all members of the Salkofflaboratory for helpful discussion and comments on this manuscript.

Correspondence should be addressed to Lawrence Salkoff, Department of Anatomy and Neurobiology, Washington University School of Medicine, 660 South Euclid Avenue, St. Louis, M0, 63110. E-mail: salkoffl@pcg.wustl.edu. DOI:10.1523/JNEUROSCI.3825-07.2008

Copyright $\odot 2008$ Society for Neuroscience $\quad$ 0270-6474/08/280757-09\$15.00/0 progressive frequency-dependent increase in outward current amplitude in response to repetitive depolarization. Thus, when two depolarizing pulses are repeated with an interpulse interval as long as $20 \mathrm{~s}$, the peak outward current evoked by the second pulse is greater than the peak current of the preceding pulse. The pattern of sequentially increasing outward current gives the impression that the availability of channels that can open during subsequent pulses is augmented by a use-dependent process occurring during the previous depolarization. We found that cumulative activation of KVS-1 is voltage and time dependent and does not depend on the influx of Ca during depolarization. The mechanism of CA is currently unknown, but a precedent for this type of channel behavior is found in some voltage-dependent calcium channels (VDCCs), which show use-dependent facilitation. Facilitation of VDCCs has been attributed to several molecular mechanisms, such as removal of G-protein inhibition, phosphorylation, and activation by calmodulin (Dolphin, 1996; Halling et al., 2006; Tedford and Zamponi, 2006). In the case of calcium channel association with G-protein, a successful model that closely simulates voltage-dependent facilitation (VDF) is based on two populations of channels, one that is associated with G-protein (and inhibited) and one that is freed from association (and uninhibited) (Boland and Bean, 1993). Using the general approach of two populations of channels, we present an analogous model of KVS-1 current behavior that closely simulates CA.

\section{Materials and Methods}

Xenopus oocyte expression. Capped KVS-1 cRNA was synthesized using the T3 mMessage mMachine kit (Ambion, Austin, TX). The KVS-1 
cDNA was kindly provided by Federico Sesti (Robert Wood Johnson Medical School, Piscataway, NJ). cRNA reactions were resuspended in nuclease-free water to a final concentration of $1.5 \mu \mathrm{g} / \mu \mathrm{l}$. Oocytes were harvested from adult female Xenopus laevis as described previously (Yuan et al., 2000). Defolliculated oocytes were injected with $\sim 75 \mathrm{ng}$ of cRNA using a Drummond Scientific (Broomall, PA) Nanojector. Injected oocytes were incubated at $18^{\circ} \mathrm{C}$ in ND96 medium (in mM): $96 \mathrm{NaCl}, 2 \mathrm{KCl}$, $1.8 \mathrm{CaCl}_{2}, 1 \mathrm{MgCl}_{2}$, and 5 HEPES, pH 7.5 with $\mathrm{NaOH}$. Oocytes were electrophysiologically analyzed 3-5 d after injection.

Electrophysiology. Xenopus oocytes. Two microelectrode voltageclamp recordings were obtained in ND96 plus 1-2 mM DIDS to block endogenous chloride conductances. For patch-clamp experiments, before recording, the vitelline membranes were mechanically removed. Cell-attached recordings were done with ND96 in the bath except as otherwise noted and with a pipette solution containing (in $\mathrm{mm}$ ) 110 $\mathrm{K}$-MES, 5 HEPES, and $2 \mathrm{KCl}, \mathrm{pH} 7.2$ with $\mathrm{KOH}$. Pipette tip resistance ranged from 1 to $2 \mathrm{M} \Omega$. Traces were acquired using an Axopatch 200A (Molecular Devices, Sunnyvale, CA), digitized at $10 \mathrm{kHz}$, and filtered at 2 kHz. Data were analyzed using pClamp 9 (Molecular Devices), SigmaPlot 8 (Systat Software, Port Richmond, CA) or Origin 6.0 (Microcal Software, Northampton, MA). Chemicals agents used in this study were purchased from Sigma (St. Louis, MO).

Transient transfected human embryonic kidney cells. Human embryonic kidney HEK-293 (HEK) cells were transiently transfected with the KVS-1 cDNA using Lipofectamine 2000 (Invitrogen, Carlsbad, CA). The cells were cultured in DMEM supplemented with $10 \%$ fetal bovine serum and penicillin-streptomycin (Invitrogen). Whole-cell patch-clamp recordings were done using 3-5 $\mathrm{M} \Omega$ pipettes filled with (in mM) $110 \mathrm{~K}-\mathrm{MES}, 10$ HEPES, 5 EGTA, and $2 \mathrm{KCl}$. The bath solution contained (in mM) $5 \mathrm{KCl}$, $140 \mathrm{NaCl}, 10 \mathrm{HEPES}$, and $2 \mathrm{CaCl}_{2}$.

Kinetic modeling. To estimate the rate constants in the kinetic scheme (see Fig. 6), the program IChMASCOT (www.ichmascot.org) was used to simultaneously fit the current traces shown in the Figure 6, $F$ and $G$. The simulations were performed in the program IChSimLab (www.ichmascot.org).

\section{Results}

\section{KVS-1 channels have greatest sequence similarity to Shab (Kv2) channels}

There are 10 families of voltage-dependent potassium channels, all of which share a common six transmembrane domain motif. One of these transmembrane domain motif (S4) contains the positive charges responsible for sensing voltage. KVS-1 channels were cloned previously and expressed in a heterologous expression system and shown to have activation and inactivation kinetics resembling potassium channels of the Shal (Kv4) family (Bianchi et al. 2003). However, a National Center for Biotechnology Information Blast comparison of the amino acid sequence of the KVS- $1 \alpha$ subunit with the $\alpha$ subunits of all 10 families of voltagedependent potassium channels showed that the KVS-1 channel was most similar in sequence to the Shab (Kv2) family. Figure $1 \mathrm{~A}$ shows an amino acid sequence alignment of KVS-1 (middle sequence) with the mouse Shab (Kv2.1) sequence (top) and the mouse Shal (Kv4.1) sequence (bottom). As indicated in Figure 1, there is significantly more sequence identity with the Shab sequence than the Shal sequence. Although the KVS-1 channel has higher sequence identity to the Shab sequence, there are a few residues present that are typical of Shal channels, which suggest that KVS-1 may be a mosaic of the Shab and Shal subfamilies. The KVS-1 gene is one of six genes in the C. elegans genome encoding paralogs that are homologous and distantly related to the Shab (Kv2) family (Wei et al., 1996) (Salkoff laboratory, unpublished results). A similar extended family of Kv2-related genes is conserved in the mammalian genome, in which seven Shab-related genes are present (Kv6.1, 6.2, 6.3; Kv8.1; Kv9.1, 9.2, 9.3). A phylogenetic tree showing the relationship of these mammalian
A

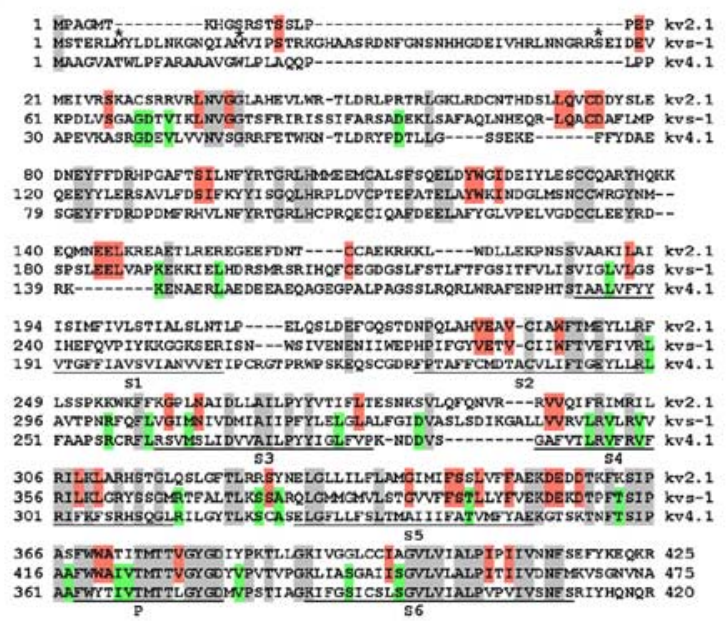

B

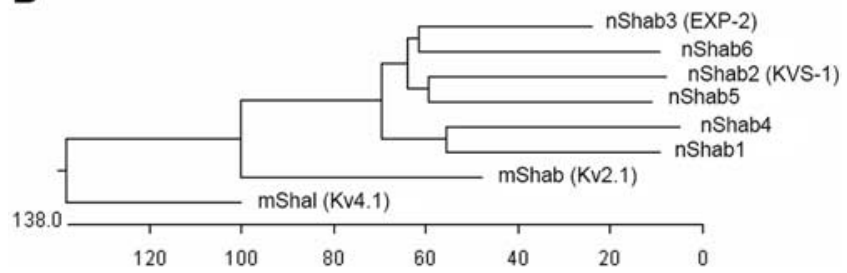

Figure 1. KVS-1 has significantly more sequence identity with Shab (Kv2) than Shal (Kv4).A, Amino acid sequence alignment of KVS-1 (middle sequence) with the mouse Shab (Kv2.1) sequence (top) and the mouse Shal (Kv4.1) sequence (bottom). In red shading are 46 residues shared by KVS-1 and Kv2.1 that are typical of Shab channels from all species and not found in Shal family channels. In contrast, green shading indicates 27 residues shared with the Kv4.1 sequence that are typical of the Shal family and are not found in the Shab family. Gray shading indicates 80 residues that are found in all three channels. Asterisks indicate sites of initiation methionines introduced by site-directed deletion constructs (see Results). $\boldsymbol{B}$, Phylogenetic tree showing that the six members of nematode nShab (Kv2) family are closer to mouse mShab (Kv2.1) than to mouse mShal (Kv4.1). Phylogenetic tree was created using the Clustal W method. Branch length corresponds to sequence divergence (horizontal axis shows relative divergence).

genes to the Kv2 family is shown in the IUPHAR Compendium of Voltage-Gated Ion Channels (Catteral et al., 2002). Conversely, in C. elegans, only one gene encodes a Shal (Kv4) type of channel (Wei et al., 1996). In a phylogentic tree, all six C. elegans Shab paralogs group together, and this grouping shows a closer relationship to the mouse Shab (Kv2.1) channel than the mouse Shal $(\mathrm{Kv} 4.1)$ channel (Fig. $1 \mathrm{~B}$ ). Thus, KVS-1 is more closely related to Shab (Kv2) channels than Shal (Kv4) channels.

\section{KVS-1 channels undergo CA}

Like Shal (Kv4) channels, KVS-1 channels exhibit quickly activating and quickly inactivating outward currents in response to a family of depolarizing step pulses. This fast transient behavior of a potassium current was first observed in molluscan neurons (Connor and Stevens, 1971) but has since been seen in almost all species. Figure $2 \mathrm{~A}$ shows a family of KVS-1 currents expressed in Xenopus oocytes and evoked by depolarizing step pulses to progressively more positive voltages using two-electrode voltage clamp (TEV). In comparison, Figure $2 B$ shows a family of currents from the C. elegans Shal (Kv4) ortholog (nShal) (Fawcett et al., 2006). These two families of currents resemble each other with regard to their fast activation and rapid inactivation. However, major differences in the behavior of these channels appear 

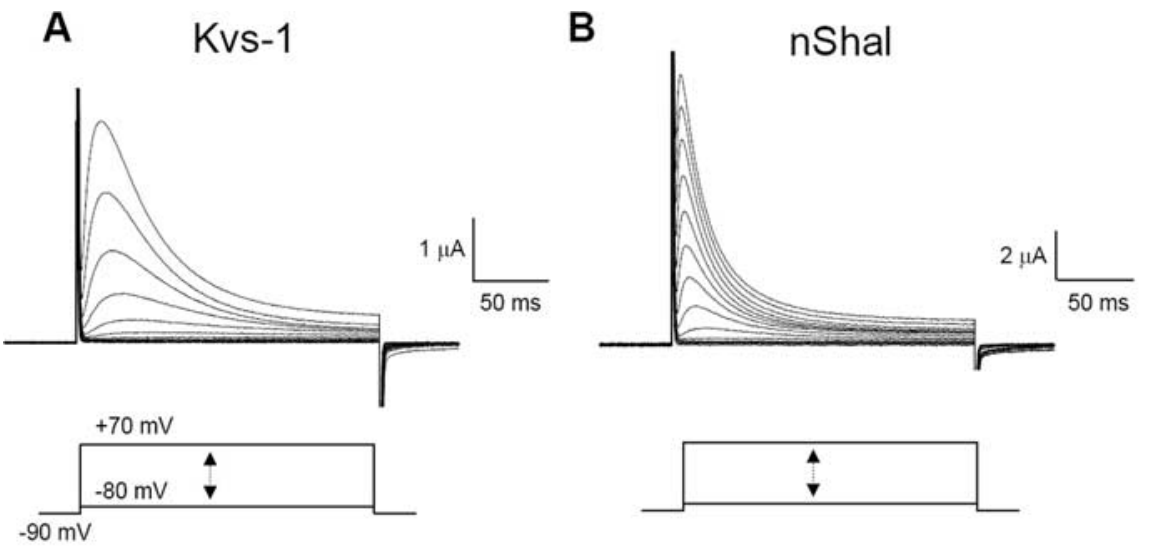

C

D
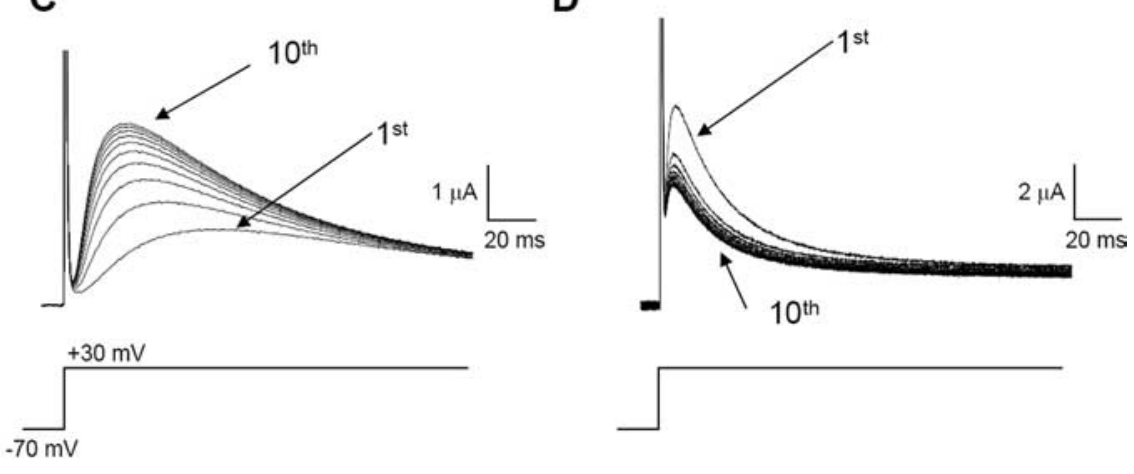

E $+50 \mathrm{mv}$
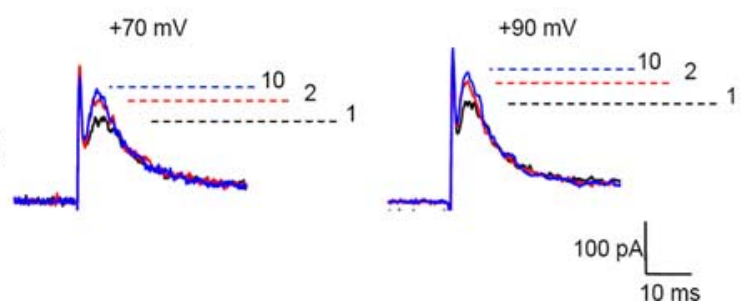

Figure 2. KVS-1 channels undergo cumulative activation in Xenopus 0ocytes. $\boldsymbol{A}$, KVS-1 current family evoked by $200 \mathrm{~ms}$ intervals to avoid the effects of (A). $\boldsymbol{B}$, nShal current family evoked by the same voltage protocol shown in $\boldsymbol{A}$. $\boldsymbol{C}$, KVS-1 currents in response to 10 identical repeated $200 \mathrm{~ms}$ pulses from -70 to $+30 \mathrm{mV}$ at $2 \mathrm{~Hz}$. Notice that peak current increases in magnitude in successive pulses. $\boldsymbol{D}$, nShal currents in response to the same protocol shown in $\boldsymbol{C}$. Notice the decrease in current magnitude in successive pulses. $\boldsymbol{E}$, KVS-1 currents in cell-attached patch-clamp experiments in response to a repeated depolarizing voltage pulse. Dashed lines indicate current levels for the 1st, 2nd, and 10th sweeps. Step pulses were to indicated (millivolts) values. The pipette contained $110 \mathrm{~mm} \mathrm{~K}^{+}$, as well as the extracellular medium; the internal voltage was thus assumed to be $\sim 0 \mathrm{mV}$. depolarizing steps from -80 to $+70 \mathrm{mV}$ in $10 \mathrm{mV}$ increments from a holding of $-90 \mathrm{mV}$ (current pulses were applied at $30 \mathrm{~s}$

\section{CA depends on the frequency of the} depolarizing step pulse

To investigate the effect of the stimulation frequency on the extent of CA, we repetitively stimulated the channels at several frequencies with the same depolarizing step pulse (Fig. 3A). In these experiments, we used $200 \mathrm{~ms}$ pulses that were long enough to activate and almost completely inactivate the channels. The extent of CA increases as the stimulation frequency increases, as measured by the fractional increase in current relative to the first pulse (Fig. $3 A-C$ ). Furthermore, Figure $3 A$ also illustrates that the absolute amplitude of current attained at the end of the series repetitive pulses is greater at the higher frequency. In addition, the process of CA occurs faster at higher frequencies. This is seen in the decrease in time necessary to reach the maximum steady-state value (Fig. $3 B, D$ ).

\section{CA is voltage dependent}

To study the effects of voltage on CA, voltage-clamp command pulses were repetitively applied at different test voltages but at a constant pulse duration and frequency (Fig. 3E). The pulse frequency chosen was $2 \mathrm{~Hz}$, because CA is robust at that frequency. By comparing fractional increases in current rather than absolute current values, we were able to observe the relative increase in current elicited at each voltage. Fractional current increased as a function of command voltage plots as a bell-shaped curve with a maximum at +30 $\mathrm{mV}$ (Fig. 3F). In these experiments, fractional current increases exponentially to reach a maximum steady-state level (Fig. $3 G)$. The time constant of fractional current increase is voltage dependent and decreases with increasing voltage (Fig. $3 H$ ). Thus, with repetitive depolarizing step pulses, the process underlying CA follows a simple exponential time course and is voltage dependent.

when currents from each are evoked by command voltage step pulses that are repeated at the same voltage (Fig. $2 C, D$ ). Figure $2 C$ shows that repetition of identical $200 \mathrm{~ms}$ pulses at a frequency of $2 \mathrm{~Hz}$ produces a more than twofold increase in peak current in KVS-1. In marked contrast, a similar repetition of identical voltage step pulses produces a progressively smaller transient outward current in Xenopus oocytes expressing nShal channels (Fig. $2 D$ ). This behavior in nShal is typical of channels of the Kv4 family, as well as other inactivating potassium channels (Aldrich et al., 1979; Marom and Levitan, 1994; Bertoli et al., 1996; Klemic et al., 1998; Eghbali et al., 2002), and can be explained by a lack of recovery from inactivation during the interval between depolarizing step pulses. KVS-1 channels apparently recover from inactivation rapidly, and, furthermore, additional channels are recruited into a readily openable state after a depolarizing step pulse. In response to repetitive depolarizing step pulses, this CA can also be seen in cell-attached patches (Fig. 2E), as well as in whole-cell currents.

\section{CA depends on the duration of the voltage step pulse} $\mathrm{CA}$, voltage-clamp command pulses were repetitively applied at a constant voltage and frequency, at three different pulse durations (Fig. 4A). In these experiments, we observed that the magnitude of the fractional current increase gets larger as the duration of the repeated step pulse increases. In addition, the absolute peak amplitude of the final level of the current gets larger as a function of the duration of the repeated step pulse. Figure $4 A$ shows that the peak amplitude of the current elicited by the first step pulse in each pulse train was approximately the same in all experiments, regardless of the pulse duration, whereas the last voltage step pulse in a train elicited greater peak current amplitude as the duration of the step pulse increased. To see the pulse duration required to elicit the maximal current amplitude, we performed the experiment shown in Figure $4 B$. In this experiment, we applied a prepulse of varied duration followed by a test pulse at a
To study the effects of the duration of the voltage step pulse on 

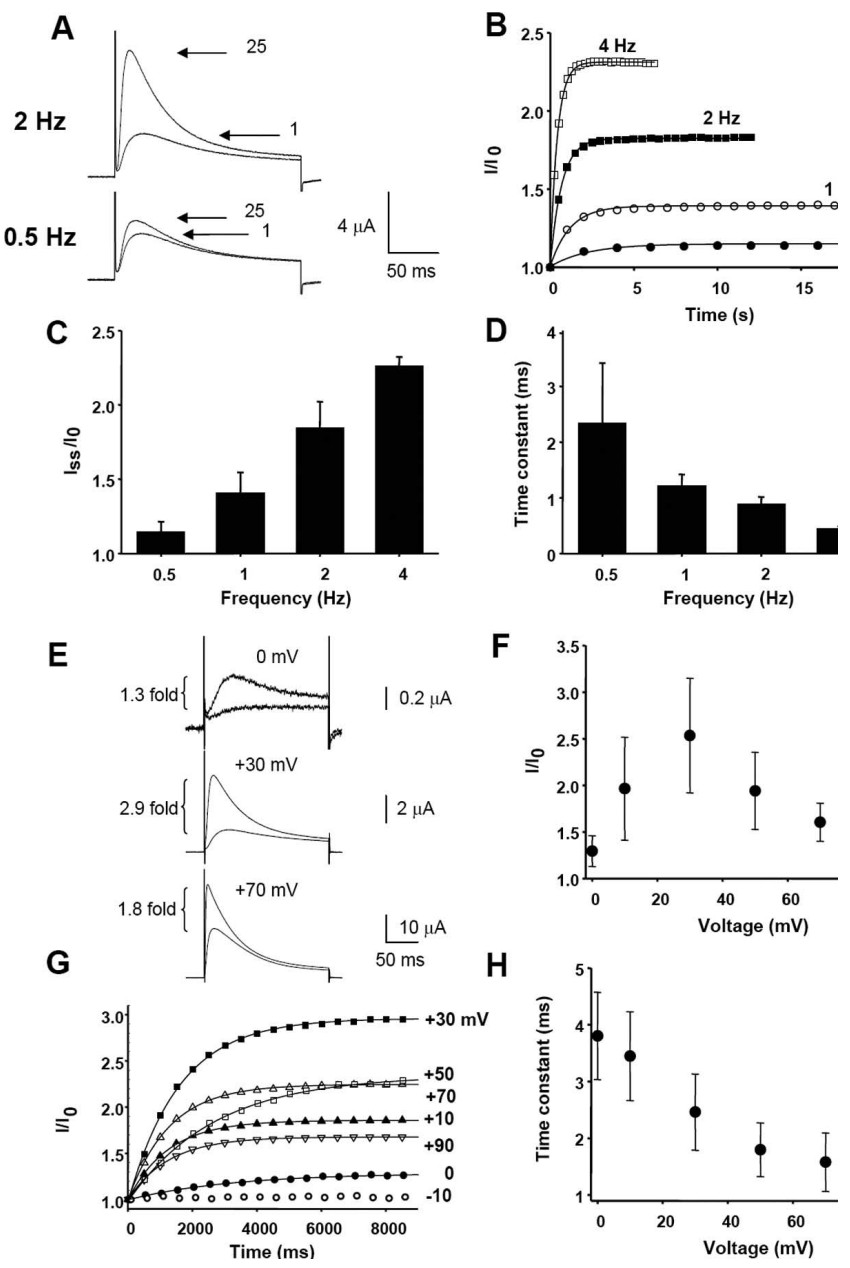

Figure 3. Frequency and voltage dependence of cumulative activation. $\boldsymbol{A}$, Repetitive $200 \mathrm{~ms}$ pulses at indicated frequencies evoke different extents of cumulative activation. A step pulse to $+30 \mathrm{mV}$ was chosen for this experiment because, as will be shown in $F$, maximal cumulative activation is evoked at this voltage. Arrows indicate the 1 st and 25 th sweeps. $\boldsymbol{B}$, Fractional increase in current depends on stimulation frequency. Peak current for each trace, elicited by repetitive $200 \mathrm{~ms}$ pulses from -70 to $+30 \mathrm{mV}$ at several frequencies (as shown in $A$ ), are shown normalized to the peak current of the first pulse. $C$, Fractional increase in current obtained at steady state $\left(I_{S S} / I_{0}\right)$, shown as mean \pm SD for several frequencies. $D$, Time constants of fractional increase in current as a function of stimulation frequency obtained by fitting points in $\boldsymbol{B}$ to single-exponential functions. The mean \pm SD is shown for the various frequencies. $\boldsymbol{E}$, First and 25 th trace for voltage pulses from -70 to $0 \mathrm{mV}$ (top), $+30 \mathrm{mV}$ (middle), and $+70 \mathrm{mV}$ (bottom) (2Hz). $\boldsymbol{F}$, The steady-state fractional increase in current as a function of voltage is bell-shaped with a maximum at $+30 \mathrm{mV}$. The mean \pm SD is shown. $\mathbf{G}$, Time course of current increase elicited by repetitive $200 \mathrm{~ms}$ pulses at indicated voltages from a voltage holding of $-70 \mathrm{mV}$ and at a frequency of $2 \mathrm{~Hz}$. Data are from same experiment as in $\boldsymbol{E}$. Each symbol represents peak current for all 25 sweeps at each voltage; solid lines are fits to single exponentials. $\boldsymbol{H}$, Time constants obtained from single-exponential fits of fractional increases in current, as a function of voltage. Shown are mean and SD.

fixed voltage and duration. The plot in Figure $4 C$ shows the absolute amplitude of the test pulse plotted as a function of the duration of the prepulse. Notably, as is evident in Figure $4 B$, although the current of the prepulse inactivates almost completely within the first $200 \mathrm{~ms}$, the continued depolarization after inactivation continues to produce an increase in the CA as measured by the test pulse. In fact, the enhancement of CA resulting from increasingly longer prepulses reaches saturation only after several seconds (Fig. 4C). Thus, long depolarizing step pulses appear to slowly recruit KVS-1 channels into a unique closed state that is rapidly convertible to a readily openable state during repolarization. By changing the voltage level of the prepulse, we see that saturation occurs faster as the prepulse voltage increases (Fig. 4C). In this figure, the time course of the onset of CA was quantified by fitting the increasing peak current amplitude evoked by the test pulse to a single-exponential function. The time course of the onset of CA is faster as voltage becomes more positive (Fig. $4 D$ ). Thus, bringing the maximum number of channels into the activated (readily openable) state depends on the length of the depolarizing pulse and its voltage. Also, it appears unlikely that all channels must transit through an open state to reach this special state. Evidence for this is that this cumulatively activated readily openable state appears to continue to be populated after initial activation and inactivation are complete and that saturation of CA is not achieved until several seconds after the onset of the depolarizing prepulse.

\section{Removal of CA during hyperpolarization}

CA accumulates with maintained depolarization and reverses with maintained hyperpolarization. The reversal of CA is time dependent and occurs at negative voltages but has only a slight voltage dependence. To measure the time required for the reversal of CA, we applied a prepulse of sufficient duration and voltage to saturate the CA effect $(2.5 \mathrm{~s}$ at $+50 \mathrm{mV})$, followed by test pulses at $+50 \mathrm{mV}$ after an interpulse of varied duration (Fig. $4 E$ ). The reversal of CA was shown by the diminishing peak amplitude of the test pulse over time. CA decays exponentially with a time constant of $\sim 2 \mathrm{~s}$ (Fig. $4 F$ ). With longer prepulses, CA was not completely removed even at $20 \mathrm{~s}$, suggesting that there may be a slower component involved in the removal of CA. Unlike the onset of CA, which is highly voltage dependent (Fig. 4A-D), the removal of CA is relatively insensitive to voltage, at least within the voltage range of -30 to $-70 \mathrm{mV}$ (Fig. $4 F, G$ ).

\section{CA is absent in a mammalian cell line}

To gain a better understanding of the CA process, KVS-1 channels were transiently expressed in HEK-293 cells. Although macroscopic currents elicited by families of voltage step pulses were fairly similar in both HEK cells and Xenopus oocytes (Fig. 5A,B), no cumulative activation was observed in HEK cells under voltage clamp in whole-cell patch-clamp configuration when subjected to repetitive depolarizing step pulses to $+30 \mathrm{mV}$ applied at $2 \mathrm{~Hz}$ (Fig. 5C). Note that, in this experiment, recovery from inactivation appears to be virtually complete after each pulse. This is in marked contrast to what is observed for the nShal (Kv4) current in response to the identical experimental protocol, in which recovery from inactivation is incomplete and, thus, evoked peak current shows a sequential decline in amplitude (Fig. 2D). The properties of KVS-1 currents observed in HEK cells are in marked contrast to those observed in Xenopus oocytes in which there is extensive CA (Fig. 5D). Interestingly, the rapid kinetics of KVS-1 currents expressed in HEK cells were similar to those observed in Xenopus oocytes after having undergone CA (Fig. 5C$F$ ). In addition to the kinetics, the activation curve of KVS-1 currents observed in HEK cells was similar to that observed in Xenopus oocytes but only after the oocyte KVS-1 activation curve was measured in the state of complete CA. This can be seen in Figure $5 F$, which shows two activation curves for KVS-1. The left-shifted activation curve was obtained after application of a depolarizing prepulse (voltage paradigm shown in Fig. 5G) and resembles that of the KVS-1 current expressed in HEK cells (Fig. $5 E$ ). The midpoint of activation for the left-shifted curve was $67 \pm 5 \mathrm{mV}$ compared with $91 \pm 8 \mathrm{mV}$ for the KVS-1 activation curve obtained in Xenopus oocytes before CA; in contrast, the 

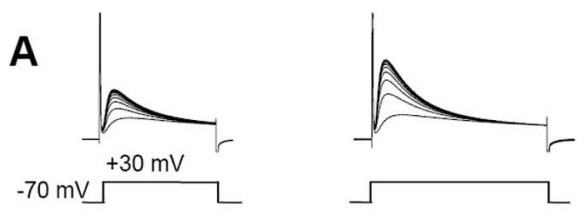

B
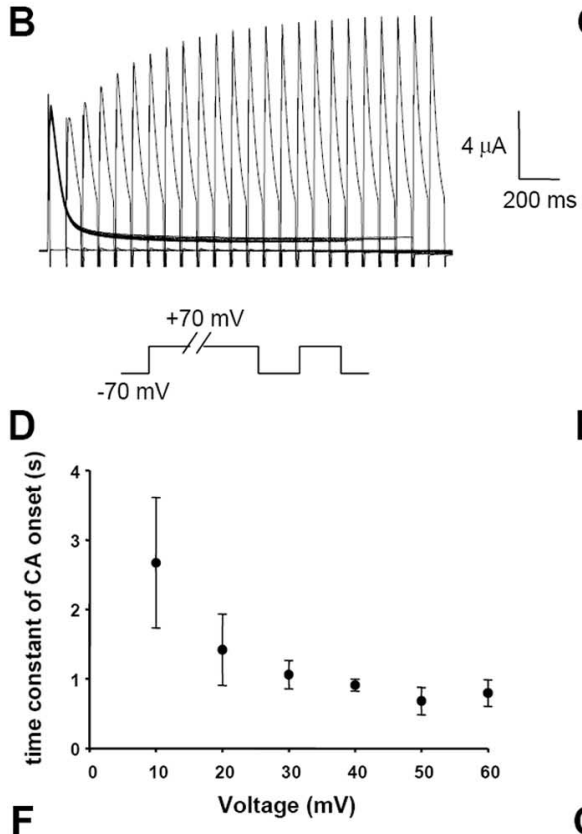

F

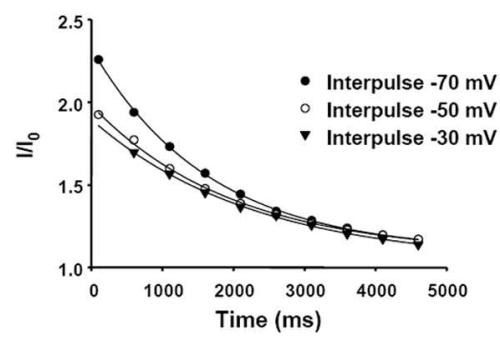

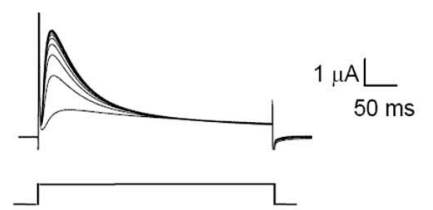

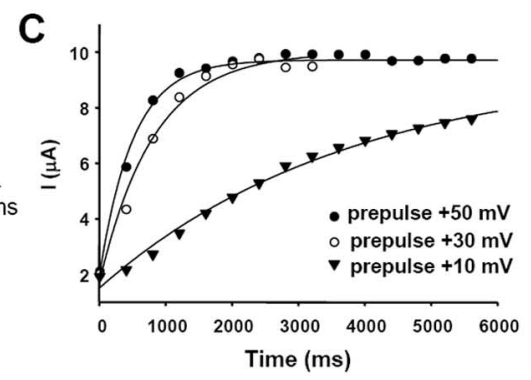

E
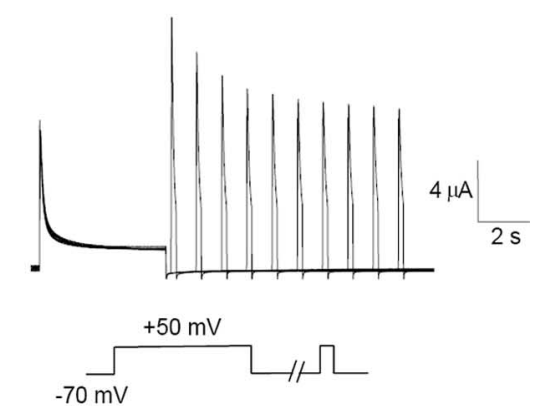

G

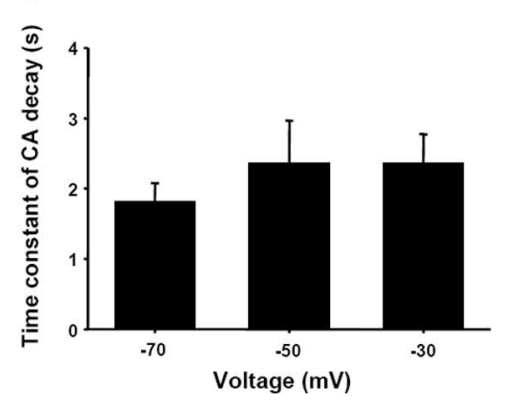

Figure 4. Cumulative activation is a time-dependent process. $A$, The extent of the fractional increase in current depends on pulse duration. Ten traces are shown of $200 \mathrm{~ms}$ (left), $300 \mathrm{~ms}$ (middle), and $400 \mathrm{~ms}$ (right) pulses from $-70 \mathrm{mV}$ to $+30 \mathrm{mV}$, at a stimulation frequency of $2 \mathrm{~Hz}$. B, Time course for the onset of CA. Prepulses of increasing duration at $+70 \mathrm{mV}$, followed by 100 ms test pulses to $+70 \mathrm{mV}$ (after a $100 \mathrm{~ms}$ interpulse at $-70 \mathrm{mV}$ ) reveal an exponential rise in test pulse current. Note that the time course for the onset of CA is slower than the time course of inactivation. $\boldsymbol{C}$, Time course of cumulative activation onset as a function of prepulse duration at different voltages. Shown are prepulses to $+50 \mathrm{mV}$ (filled circles), $+30 \mathrm{mV}$ (open circles), and $+10 \mathrm{mV}$ (filled triangles). Solid lines are fit to single-exponential functions. $\boldsymbol{D}$, Time constants of cumulative activation onset as a function of prepulse voltage, obtained from single-exponential fits from two pulse protocols as in $\boldsymbol{C}$. Shown are means \pm SD. $\boldsymbol{E}$, Two pulse protocol to study CA decay. 2.5 s prepulses from -70 to $+50 \mathrm{mV}$ are followed by an interpulse of varied duration; the $100 \mathrm{~ms}$ test pulse is to $+50 . \boldsymbol{F}$, Time course of CA decay at several interpulse voltages: -70 (filled circles), -50 (open circles), $-30 \mathrm{mV}$ (filled triangles). Solid lines are fits to single-exponential functions. $\mathbf{G}$, Time constants of CA decay obtained from exponential fits as shown in $\boldsymbol{F}$ are shown for different voltages. Time constants are weakly voltage sensitive. Shown are mean \pm SD.

midpoint of activation for the KVS-1 current expressed in HEK cells was $81 \pm 7 \mathrm{mV}$. Note that the $G-V$ curve obtained after CA had a higher absolute maximum conductance compared with the control curve in which no depolarizing prepulse was applied, attributable probably to an increase in the number of active channels. These curves were fitted with the sum of two Boltzmann functions, each representing one of two presumed channel populations (representing channels before and after CA; see below) and permitting only the amplitude of each component to vary. One interpretation of these results is that the channels in HEK cells are "locked" in a state analogous to the CA state in Xenopus oocytes in which CA is maximal. We discuss this again later when we show that the kinetics of the KVS-1 current in HEK cells closely resembles that of the KVS-1 current expressed in Xenopus oocytes after reaching the state of complete CA. Discrepancies between Xenopus oocytes and HEK cells have been reported previously for other ion channels, in which a possible explanation is the existence of a soluble factor that is absent in whole-cell patch-clamp experiments attributable to cell dialysis (Varela et al., 2002). To address this possibility, whole-cell voltage-clamp experiments were performed using the perforated patch technique using the antibiotic nystatin, but CA was still not observed (data not shown).

Although these results could conceivably be attributable to an esoteric property of Xenopus oocytes, the track record of Xenopus oocytes revealing conserved aspects of channel behavior typical of differentiated cells is rather good. This is apparently because Xenopus oocytes contain many conserved products that are found later in development in subsets of differentiated cells. One example of this is the discovery of the intrinsic mechanism of rectification of mammalian inward rectifier potassium channels. In that case, the presence of polyamines in the Xenopus oocyte caused the strong inward rectification characteristic of the mammalian inwardly rectifying potassium channel (IRK) in native cells (Lopatin et al., 1994). Another example is the discovery of the heteromeric assembly of native $I_{\mathrm{KS}}$ potassium channels from KCNQ1 $\alpha$ subunits and KCNE1 accessory subunits. We now understand that native channels in cardiac myocytes are composed of these two subunits. However, expression of KCNQ1 in mammalian cell lines (which lack KCNE1) produced currents substantially differing in kinetic properties from those seen when KCNQ1 is expressed in Xenopus oocytes. This paradoxical result was resolved by the realization that mammalian KCNQ1 channel subunits associated with endogenous KCNE1 Xenopus subunits present in the oocytes. Thus, expression of KCNQ1 subunits in Xenopus oocytes produced a current that more closely matched the current in native cells than expression of KCNQ1 subunits in mammalian cell lines (Sanguinetti et al., 1996).

\section{Voltage range of CA}

Although the voltage range of activation of KVS-1 channels is quite positive, the KVS-1 current and CA can be seen with depolarizing pulses to $0 \mathrm{mV}$ (Fig. $3 E$ ). In general, however, there is evidence that much of the electrical activity in C. elegans is shifted to positive values relative to mammalian cells, and many of the voltage-dependent ion channels in C. elegans function in a more 
positive voltage range than their mammalian counterparts. We showed this to be true for the Shaker (Kv1) and Shal (Kv4) channels (Fawcett et al., 2006) and Shaw (Kv3) EGL-36 channels (Johnstone et al., 1997). In fact, an egl-36 mutation that shifted the voltage dependence of activation of Shaw EGL-36 channels to more negative voltages typical of mammalian channels resulted in excessively active Shaw EGL-36 channels producing the egl phenotype.

\section{Role of $\mathrm{N}$ terminus}

Inactivation processes in potassium channels are mediated by either an intracellular N-terminal "inactivation ball” peptide (Ntype inactivation) (Hoshi et al., 1990) or conformational changes in the pore region (P/C-type inactivation) (Callahan and Korn, 1994; Liu et al., 1996; Starkus et al., 1997). To investigate whether KVS-1 is inactivated by a classical N-type mechanism and whether this plays a central role in CA, we created $\mathrm{N}$-terminal deletions so that translation would be initiated at either methionine 7 or 18 and also created a larger deletion, adding an initiator methionine at position 53 (Fig. 1). None of these three $\mathrm{N}$-terminal alterations eliminated fast inactivation, and only slight alterations in gating kinetics resulted (data not shown). Significantly, CA was present in currents produced by all three constructs. To further investigate the role of rapid inactivation on CA, we injected oocytes expressing wild-type KVS currents with tetraethylammonium (TEA). In some of these experiments, inactivation was slowed by a factor of 10 relative to KVS currents in oocytes not injected with TEA. Nevertheless, CA was present under all circumstances (data not shown). We should note that, although N-terminal deletions as described above failed to remove inactivation in our experiments, recent work done in $\mathrm{CHO}$ cells reports that a 40 amino acid N-terminal deletion primarily abolished rapid inactivation (Cai and Sesti, 2007). We have no explanation for this discrepancy except to point out that our work was done in the Xenopus oocyte expression system whereas their work was undertaken in $\mathrm{CHO}$ cells, which may be a factor in these different results. In Shaker potassium channels, external TEA has been shown to slow P-type inactivation by obstructing the pore and preventing its collapse (Choi et al., 1991). However, we found that external TEA failed to alter the kinetics of inactivation of wild-type KVS-1 channels (data not shown).

\section{CA may be attributable to a use-dependent modification of channels}

In Figure 6, we analyzed CA in KVS-1 channels assuming that CA might be attributable to two populations of channels, a preacti-
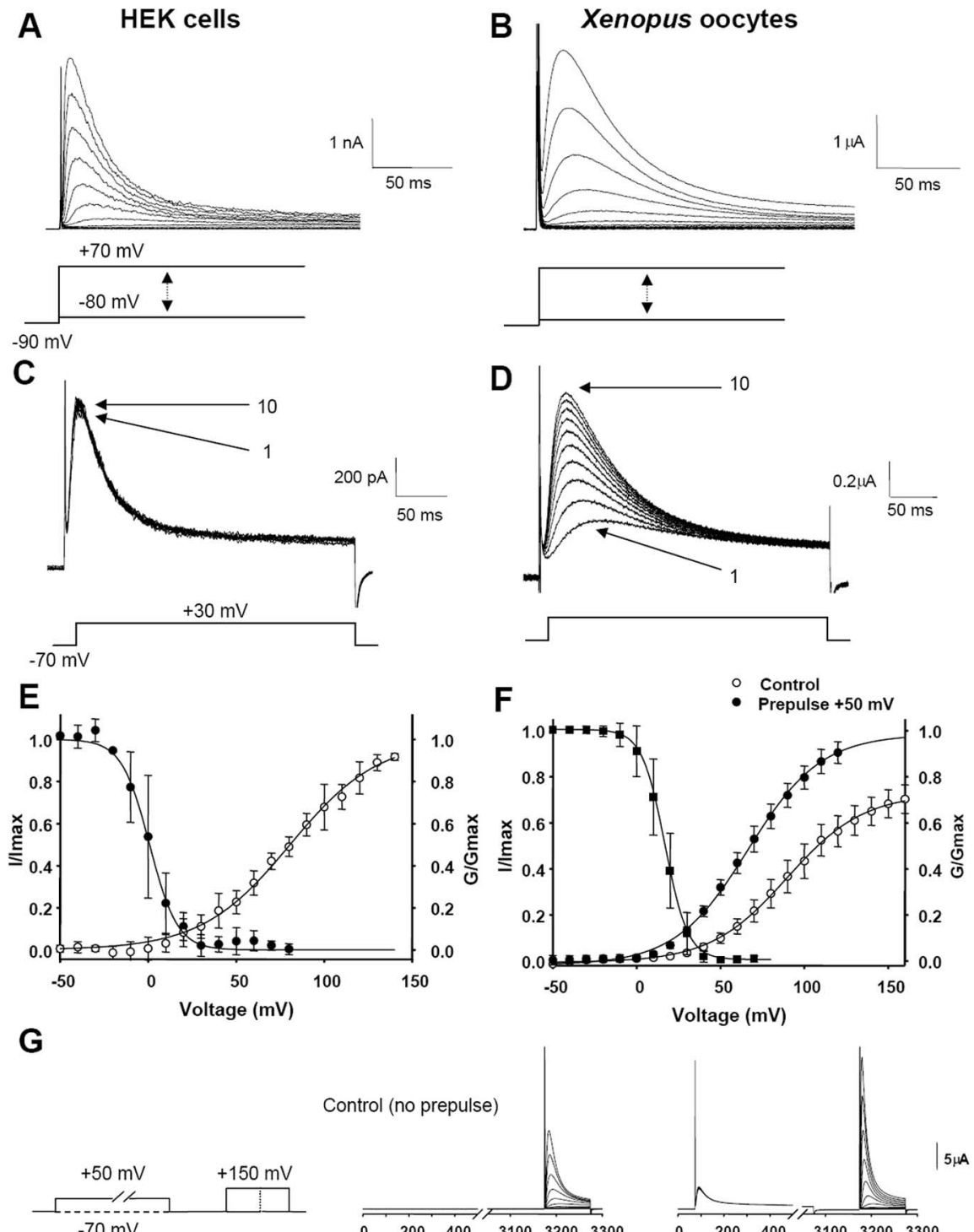

Figure 5. Cumulative activation is present in Xenopus oocytes but not in HEK cells. $A$, A family of KVS-1 currents recorded from transiently transfected HEK-293 cells in whole-cell patch-clamp configuration in response to voltage steps between -80 and $+70 \mathrm{mV}$ in $10 \mathrm{mV}$ increments from a holding of $-90 \mathrm{mV}$. $\boldsymbol{B}$, KVS-1 currents recorded from Xenopus oocytes using TEV and the same pulse protocol as in $\boldsymbol{A}$. C, Cumulative activation does not occur in transiently transfected HEK-293 cells in response to repeated $(2 \mathrm{~Hz}) 200 \mathrm{~ms}$ depolarizing pulses to $+30 \mathrm{mV}$. Arrows indicate the 1st and 10th pulse. $D$, Cumulative activation is present in KVS-1 expressed in Xenopus oocytes in response to the same pulse protocol in C. First and 10th pulses are indicated by arrows. $\boldsymbol{E}$, Steady-state activation (open circles) and inactivation (filled circles) for KVS-1 currents in transfected cells. Solid lines indicate fits to Boltzmann function. Activation curve was fitted a Boltzmann function with the following values: $V_{\mathrm{m}}=80.67 \pm$ $6.7, k=25.2 \pm 3.8$ Inactivation curve was fit with a Boltzmann function with the same values: $V_{\mathrm{m}}=0.5 \pm 7.4, k=6.5 \pm 0.8$. $\boldsymbol{F}$, Steady-state activation (circles) and inactivation (squares) for KVS-1 currents in Xenopus 0ocytes; steady-state activation is shown in the presence (filled circles) or absence (open circles) of a $2.5 \mathrm{~s}, 50 \mathrm{mV}$ prepulse. Solid lines are Boltzmann fits. Activation curves were fitted to the sum two Boltzmann functions, with the following values: $V_{\mathrm{m} 1}=95.5 \pm 3.06, V_{\mathrm{m} 2}=50.18 \pm 2.02, k_{1}$ $=21.38 \pm 4.25, k_{2}=14.47 \pm 1.69 ;$ control curve $A_{1}=0.65 \pm 0.09, A_{2}=0.08 \pm 0.09 ;$ cumulative activated curve $A_{1}=$ $0.54 \pm 0.09, A_{2}=0.51 \pm 0.09$. Inactivation curve was with a Boltzmann function with the same values: $V_{\mathrm{m}}=16.8 \pm 5.2, k_{1}$ $=6.5 \pm 0.8$. G, Current traces in Xenopus 00cytes obtained with (right) or without (middle) a 2.5 -s-long prepulse to $+50 \mathrm{mV}$ applied before each voltage step pulse (left)

vated population and a cumulatively activated population, which have been modified by a use-dependent process. This hypothesis is similar to one used to explain the use-dependent facilitation for calcium channels (Boland and Bean, 1993). In a train of identical depolarizing pulses, KVS-1 currents evoked in response to the first and last pulse differ dramatically with respect to their kinetics. This is most evident when the amplitude of currents from the 
A

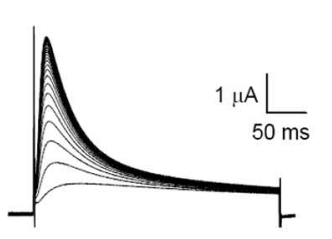

B

C
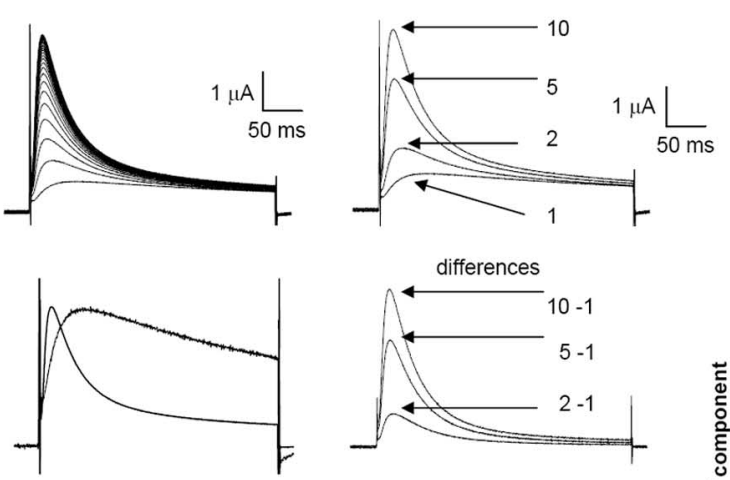

D

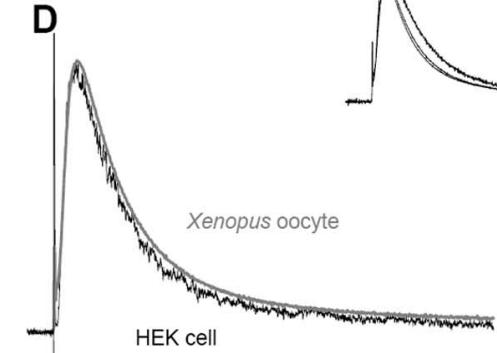

HEK cell

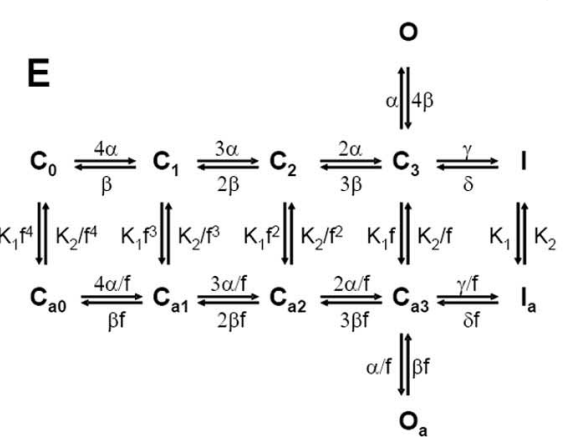

$\mathbf{F}$

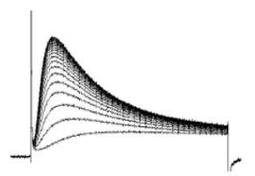

G
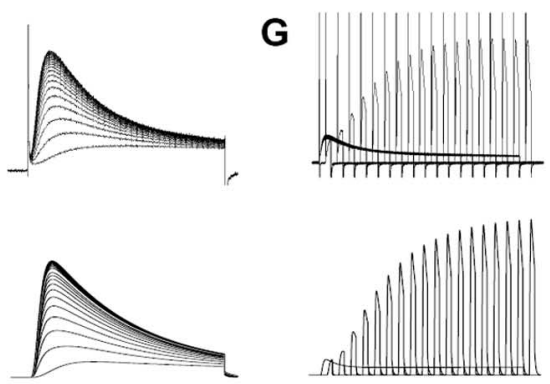

Figure 6. Two populations of channels are present in CA. $A$, Currents elicited in response to 25 repeated step pulses ( $200 \mathrm{~ms}$, $+30 \mathrm{mV}, 2 \mathrm{~Hz}$ ) (top). The first and 25th sweeps were normalized to maximum peak current (bottom). $\boldsymbol{B}$, First, 2nd, 5th, and 10th sweeps from $A$, indicated by arrows (top). Subtracting the first trace from each subsequent trace yields the traces shown (middle). During normalization to peak current amplitude, these traces show nearly identical waveforms (bottom), which are similar in having rapid inactivation and notably distinct from the slowly inactivating waveform of the first trace, which has been subtracted out. C, Current traces from $\boldsymbol{A}$ (shown in black) were simultaneously fit with three exponentials; one for slow inactivation, one for fast inactivation (shown in gray), and a component for activation (also shown in gray). Only the amplitudes of each component were allowed to vary, without changing time constants (top). Amplitudes for each of the inactivation components are shown at the bottom. $\boldsymbol{D}$, KVS-1 current trace at $+30 \mathrm{mV}$ in HEK cell (black) compared with subtracted trace (10th, first sweep in $\boldsymbol{B}$ above) in Xenopus 0ocyte (gray), also at $+30 \mathrm{mV}$. $\boldsymbol{E}$, Simple kinetic model for KVS-1 gating. Channel gating is governed by four independent subunits, when all four subunits are in activated conformation the channel opens. Top row represents the preactivated channels before $C A$; bottom row represents cumulatively activated channels that have undergone CA (see Results). Transitions between two populations are represented by vertical lines. Rate constants (1/s) are $\alpha=2.9 \exp (1.7 \mathrm{VF} / \mathrm{RT}), \beta=31$ $\exp (-0.3 \mathrm{VF} / \mathrm{RT}), k_{1}=1.8, k_{2}=0.001, f=0.2, \gamma=2.7 \exp (1.6 \mathrm{VF} / \mathrm{RT}), \delta=13 \exp (-1.8 \mathrm{VF} / \mathrm{RT})$, where $\mathrm{V}$ is the voltage, $\mathrm{R}$ is the gas constant, $F$ is Faraday's constant, $T$ is absolute temperature. $F$, Raw data (top) and simulated current (bottom) for 300 ms repetitive pulse from -70 to $+30 \mathrm{mV}$ every $0.5 \mathrm{~s}$. G, Raw data (top) and simulated current (bottom) for a protocol with increasing duration of prepulse at $+30 \mathrm{mV}$, followed by $100 \mathrm{~ms}$ test pulses to $+30 \mathrm{mV}$ (with a 100 ms hyperpolarizing interpulse to $-70 \mathrm{mV}$ ). Protocol is similar to the one in Figure $4 B$, intended to determine the time course for the onset of $C A$.

first and last pulse are normalized (Fig. $6 \mathrm{~A}$, bottom traces). We assumed that the preactivated population of KVS-1 channels is most evident in the current evoked by the initial depolarizing step pulse, and its most distinguishing characteristic is its slow inactivation. Conversely, the cumulatively activated population of channels is most evident in the current evoked by the last depolarizing voltage pulse, and its most distinguishing characteristic is its rapid inactivation. Making these assumptions, we analyzed CA observed in trains of depolarizing step pulses by two methods. In the first method, we subtracted the current observed in response to the initial depolarizing pulse from all of the subsequent current records (Fig. 6B). Quite remarkably, all of the subsequent current traces that had the initial current removed by subtraction had quite similar inactivation kinetics that resembled the current evoked by the last voltage pulse (Fig. 6B, bottom, normalized traces). We interpret this to suggest that (1) the initial current was carried mostly by the preactivated population of channels, (2). currents evoked in response to subsequent voltage pulses were carried by a mixture of preactivated and cumulatively activated channels, and (3) currents evoked in response to subsequent voltage pulses were carried by an approximately similar number of preactivated channels but an increasing number of cumulatively activated channels. This increasing number of cumulatively activated channels is created by the use-dependent modification responsible for the CA phenomenon. Also quite notably, the current traces in Figure $6 B$ (middle and bottom) were remarkably similar to the current traces from KVS-1 expressed in HEK cells with regard to both their activation and inactivation kinetics (Fig. 6D), supporting the hypothesis that the KVS-1 channels in HEK cells behave in a virtually identical manner to KVS-1 channels from Xenopus oocytes after undergoing complete CA. In the second method of analysis (Fig. 6C), we applied a mathematical fit to all of the current traces resulting from a train of identical depolarizing pulses. Assuming two different populations of channels, current sweeps were fitted with two components of inactivation, one corresponding to the first trace and one corresponding to the last trace (Fig. $6 \mathrm{C}$ ). The time constants for these components were simultaneously fitted, with only the amplitude of each component allowed to freely vary. The slow inactivation component showed a small increase in amplitude (1.5 times), whereas the amplitude of the fast inactivation component increased 5.9 times at the 25th sweep (Fig. 6C). These values were calculated between the 25th and 2nd sweep, because the determination of parameters in the first sweep had too much uncertainty. Note that all traces were fitted with the same activation time constant, the amplitude of which increased with subsequent sweeps. The two methods of analysis described above were consistent with one another; in both methods, the preactivated population of channels (the slow component) dominates the current response in the initial traces and remains relatively constant, whereas the 
cumulatively activated population of channels (the fast component) becomes proportionately larger in subsequent sweeps. The transition between the two channel types seems to be attributable to repetitive or prolonged depolarization, which appears to convert the preactivated population of channels to a cumulatively activated form.

\section{Discussion}

In this study, we revealed the existence of a novel use-dependent phenomenon in the C. elegans KVS-1 potassium channel, which we call CA. CA is a voltage-dependent phenomenon that consists of a progressive increase in current amplitude in response to repetitive stimulation or prolonged depolarization. CA was observed in the Xenopus oocyte heterologous expression system but was totally absent in HEK cells transfected with KVS-1. The KVS-1 current expressed in Xenopus oocytes can exhibit the CA effect for as long as $20 \mathrm{~s}$ after a prolonged depolarizing prepulse.

\section{Resemblance to calcium channel facilitation}

In some voltage-dependent calcium channels, a similar usedependent phenomenon known as VDF has been described (Costantin et al., 1998). VDF, which results in an increase in current amplitude in response to large depolarizing pulses, has variously been attributed to mechanisms such as phosphorylation-dephosphorylation cycles, recovery from G-protein inhibition, and activation by $\mathrm{Ca}-$ calmodulin (Dolphin, 1996). In bullfrog sympathetic neurons, it has been proposed that channels in the resting state are inhibited by a physical interaction with G-proteins, which is removed by large depolarizing pulses producing VDF (Boland and Bean, 1993; Golard and Siegelbaum, 1993). This proposal suggests a two-channel population model consisting of an inhibited G-protein-bound population and an uninhibited G-protein-free facilitated population. Additionally, $\mathrm{VDF}$ of $\mathrm{Ca}_{\mathrm{v}} 2$ has been reported to involve $\mathrm{Ca}$-calmodulin activation, triggered by local increases in calcium concentration, which produce an increase in the open probability at the single-channel level (Halling et al., 2006).

\section{Two populations of channels}

In some respects, CA of KVS-1 channels suggests a model similar to that of calcium channels, in which the channel population can be divided into a preactivated population (as in the case of G-protein-bound calcium channels) and a cumulatively activated population (such as calcium channels liberated from G-protein contact by previous depolarization) (Boland and Bean, 1993). In the case of KVS-1 channels, the two populations of channels could be distinguished by a similar association of one population binding to an accessory subunit or G-protein or by modification of one population in a use-dependent manner, perhaps by a kinase or other covalent modifier. Evidence for two populations of channels can be seen in the repetitive pulse experiments (such as in Fig. 6A) in which the current produced by the first depolarizing pulse can be assumed to be carried predominantly by preactivated slowly inactivating channels and subsequent pulses evoking both types of channels; however, subsequent pulses evoke proportionately less preactivated channels and more cumulatively activated rapidly inactivating channels. KVS-1 currents expressed in HEK cells seem to resemble only the currents from the cumulatively activated channel population seen in Xenopus oocytes, suggesting a possibility that KVS-1 channels in HEK cells are locked into the cumulatively activated conformation producing very rapid activating and inactivating currents. In Figure 6D, we show a comparison of KVS-1 currents evoked by a command voltage to $+30 \mathrm{mV}$ in HEK cells and in
Xenopus oocytes and show that they are virtually identical with respect to their activation and inactivation kinetics [the current from oocytes was the 25th evoked in a series of identical pulses and therefore had reached the state of nearly complete CA; the current of the first trace had also been removed (see figure legend)]. This similarity strongly suggests that, in HEK cell expression, only one form of KVS channels is found, and the behavior of this channel resembles the cumulative activated population observed in Xenopus oocytes. In the case of calcium channels composed of $\alpha 1 C+\beta 3$ subunits, the addition of the $\alpha 2 \delta$ subunit eliminates facilitation but produces a current with a $G-V$ relationship that is left shifted, similar to that of fully facilitated calcium channels (Platano et al., 2000). This suggests that channels containing $\alpha 2 \delta$ subunits are also locked in the facilitated conformation, so no additional facilitation is possible. This appears to be analogous to the expression of KVS-1 channels in HEK cells.

To offer a mechanistic explanation of CA, we propose a two row kinetic model, each of which represents a distinct channel kinetic population (preactivated and cumulatively activated), which consists of four closed states with one open and one inactivated state (Fig. $6 E$ ). The four closed states represent the movement of voltage sensors from the resting position (left) to the activated position nearest the open state (right), with a voltagedependent transition between the last closed state and the open state. The rate constants that connect the states between the two rows are not voltage dependent. This model is similar to previous models proposed for calcium channel facilitation (Boland and Bean, 1993; Golard and Siegelbaum, 1993). Assuming all channels at rest are in the deepest closed state $(\mathrm{C} 0)$, the current in the first pulse is carried predominantly by channels that reach the open state along the top row of the model with slow activation and slow inactivation kinetics. During CA, channels progressively transition to the bottom row of the model, which has faster activation kinetics, resulting in increased channel opening. Actual data of CA (Fig. 6F, $G$, top traces) is closely simulated by this model (Fig. 6F, G, bottom traces). The rate constants used for this simulation are given in the legend to Figure 6. An unusual feature of this model is that channels do not enter inactivation through an open state.

\section{Cell-type specificity of cumulative activation}

The total lack of CA in KVS-1 channels expressed in HEK cells is a dramatic example of how the behavior of a channel subunit can be drastically changed by the cytoplasmic environment of a given cell. Cell-type differences in the functional properties of ion channels have been reported previously and attributed to several factors such as the presence or absence of a cofactor, accessory protein, signaling cascade, or other cytoplasmic product in one expression system versus the other. For example, the inactivation kinetics of Kv1 channels coexpressed with the Kv $\beta 3$ subunit produces currents with markedly different inactivation kinetics in different heterologous systems (Heinemann et al., 1995, 1996; Leicher et al., 1998; Bähring et al., 2004). Obviously, an unknown factor differs among different heterologous systems. KVS-1 channels have been shown to coassemble with regulatory MPS proteins (MPS1 to MPS4) in both native cells and heterologous systems, in which they affect both current amplitude and channel kinetics (Park et al., 2005). Our coexpression experiments with MPS-1, which has been shown previously to produce an inhibition of KVS-1 current and an acceleration of inactivation in $\mathrm{CHO}$ cells (Bianchi et al., 2003), confirm the effects on the current but reveal no effect on CA (data not shown). However, the effect of other MPS regulatory factors on CA remains to be determined. Conceivably, CA could also depend on a cytoplasmic diffusible 
factor as seen in strongly inward rectifying IRK1 potassium channels such as spermidine or other cytoplasmic polyamines (Lopatin et al., 1994). This observation led to the discovery of cytoplasmic polyamine-mediated intrinsic rectification, which is a common mechanism of strong inward rectification. To explain the lack of CA in HEK cells, we cannot rule out the possibility that the KVS-1 channels had a different subunit composition or that the channels were posttranslationally modified differently.

\section{Physiological role and plasticity}

KVS-1 channels are expressed in several tissues in C. elegans, including chemosensory neurons, touch-sensitive neurons and motor neurons, as well as vulva and anal depressor muscle, and mutant phenotypes include locomotion, chemosensation, and mechanosensation (Bianchi et al., 2003). If CA occurred in any of these cell types, it would produce an increase in potassium current after depolarizing electrical activity. However, the physiological implications of CA are unknown attributable to our limited knowledge of the electrical activity in the neurons and muscle cells of C. elegans. However, use-dependent increases or decreases in potassium conductance have been shown to be involved in models of simple learning such as behavioral sensitization (Seigelbaum et al., 1982). CA could also have profound effects on various fundamental neurophysiological processes such as patterned neural output and neurotransmitter release. CA of KVS-1 channels, if present at presynaptic neuron terminals, would be a good candidate for a mechanism of short-term synaptic depression of neurotransmitter release, because repetitive depolarizations would result in progressively smaller depolarizing potentials, less calcium entry, and thus less neurotransmitter release. In mammals, synaptic depression is common in the CNS and may contribute to many signal processing functions such as temporal integration and coincidence detection (Cook et al., 2003). In $C$. elegans, some motor neurons also undergo synaptic depression (Wang et al., 2001), and KVS-1 may be present in the same neurons (Bianchi et al., 2003). It thus remains to be determined in $C$. elegans whether CA in KVS-1 channels plays a role in the associated mutant phenotypes of locomotion chemosensation and mechanosensation and whether CA is a conserved mechanism also found in mammals.

\section{References}

Aldrich RW, Getting PA, Thompson SH (1979) Inactivation of delayed outward current in molluscan neurone somata. J Physiol (Lond) 291:507-530.

Bähring R, Vardanyan V, Pongs O (2004) Differential modulation of Kv1 channel-mediated currents by co-expression of Kvbeta3 subunit in a mammalian cell-line. Mol Membr Biol 21:19-25.

Bertoli A, Moran O, Conti F (1996) Accumulation of long-lasting inactivation in rat brain $\mathrm{K}^{+}$-channels. Exp Brain Res 110:401-412.

Bianchi L, Kwok SM, Driscoll M, Sesti F (2003) A potassium channel-MiRP complex controls neurosensory function in Caenorhabditis elegans. J Biol Chem 278:12415-12424.

Boland LM, Bean BP (1993) Modulation of N-type calcium channels in bullfrog sympathetic neurons by luteinizing hormone-releasing hormone: kinetics and voltage dependence. J Neurosci 13:516-533.

Cai SQ, Sesti F (2007) A new mode of regulation of N-type inactivation in a Caenorhabditis elegans voltage-gated potassium channel. J Biol Chem 282:18597-18601.

Callahan MJ, Korn SJ (1994) Permeation of $\mathrm{Na}^{+}$through a delayed rectifier $\mathrm{K}^{+}$channel in chick dorsal root ganglion neurons. J Gen Physiol 104:747-771.

Catteral WA Chandy KG, Gutman GA (2002) The IUPHAR compendium of voltage-gated ion channels. Leeds, UK: IUPHAR Media.

Choi KL, Aldrich RW, Yellen G (1991) Tetraethylammonium blockade distinguishes two inactivation mechanisms in voltage-activated $\mathrm{K}^{+}$channels. Proc Natl Acad Sci USA 88:5092-5095.
Connor JA, Stevens CF (1971) Voltage clamp studies of a transient outward membrane current in gastropod neural somata. J Physiol (Lond) 213:21-30.

Cook DL, Schwindt PC, Grande LA, Spain WJ (2003) Synaptic depression in the localization of sound. Nature 421:66-70.

Costantin JL, Qin N, Zhou J, Platano D, Birnbaumer L, Stefani E (1998) Long lasting facilitation of the rabbit cardiac $\mathrm{Ca}^{2+}$ channel: correlation with the coupling efficiency between charge movement and pore opening. FEBS Lett 423:213-217.

Dolphin AC (1996) Facilitation of $\mathrm{Ca}^{2+}$ current in excitable cells. Trends Neurosci 19:35-43.

Eghbali M, Olcese R, Zarei MM, Toro L, Stefani E (2002) External pore collapse as an inactivation mechanism for Kv4.3 $\mathrm{K}^{+}$channels. J Membr Biol 188:73-86.

Fawcett GL, Santi CM, Butler A, Harris T, Covarrubias M, Salkoff L (2006) Mutant analysis of the Shal (Kv4) voltage-gated fast transient $\mathrm{K}+$ channel in Caenorhabditis elegans. J Biol Chem 281:30725-30735.

Golard A, Siegelbaum SA (1993) Kinetic basis for the voltage-dependent inhibition of N-type calcium current by somatostatin and norepinephrine in chick sympathetic neurons. J Neurosci 9:3884-3894.

Halling DB, Aracena-Parks P, Hamilton SL (2006) Regulation of voltagegated $\mathrm{Ca}^{2+}$ channels by calmodulin. Sci STKE Jan:318:er1.

Heinemann SH, Rettig J, Wunder F, Pongs O (1995) Molecular and functional characterization of a rat brain Kvb3 potassium channel subunit. FEBS Lett 377:383-389.

Heinemann SH, Rettig J, Graack HR, Pongs O (1996) Functional characterization of Kv channel b-subunits from rat brain. J Physiol 493:625-633.

Hoshi T, Zagotta WN, Aldrich RW (1990) Biophysical and molecular mechanisms of Shaker potassium channel inactivation. Science 250:533-538.

Johnstone DB, Wei A, Butler A, Salkoff L, Thomas JH (1997) Behavioral defects in C. elegans egl-36 mutants result from potassium channels shifted in voltage-dependence of activation. Neuron 19:151-164.

Klemic KG, Shieh CC, Kirsch GE, Jones SW (1998) Inactivation of Kv2.1 potassium channels. Biophys J 74:1779-1789.

Leicher T, Bähring R, Isbrand D, Pongs O (1998) Coexpression of the KCNA3B gene product with Kv1.5 leads to a novel A-type potassium channel. J Biol Chem 273:35095-35101.

Liu Y, Jurman ME, Yellen G (1996) Dynamic rearrangement of the outer mouth of a K ${ }^{+}$channel during gating. Neuron 16:859-867.

Lopatin AN, Makhina EN, Nichols CG (1994) Potassium channel block by cytoplasmic polyamines as the mechanism of intrinsic rectification. Nature 372:366-369.

Marom S, Levitan IB (1994) State-dependent inactivation of the Kv3 potassium channel. Biophys J 67:579-589.

Park KH, Hernandez L, Cai SQ, Wang Y, Sesti F (2005) A family of K+ channel ancillary subunits regulate taste sensitivity in Caenorhabditis elegans. J Biol Chem 280:21893-21899.

Platano D, Qin N, Noceti F, Birnbaumer L, Stefani E, Olcese R (2000) Expression of the alpha(2)delta subunit interferes with prepulse facilitation in cardiac L-type calcium channels. Biophys J 78:2959-2972.

Sanguinetti MC, Curran ME, Zou A, Shen J, Spector PS, Atkinson DL, Keating MT (1996) Coassembly of KvLQT1 and mink (IsK) proteins to form cardiac $\mathrm{I}_{\mathrm{KS}}$ potassium channels. Nature 384:80-83.

Seigelbaum SA, Camargo JS, Kandel ER (1982) Serotonin and cyclic AMP close single potassium channels in Aplysia sensory neurones. Nature 299:413-417.

Starkus J, Kuschel L, Rayner MD, Heinemann S (1997) Ion conduction through C-type inactivated Shaker channels. J Gen Physiol 110:539-550.

Tedford HW, Zamponi GW (2006) Direct G protein modulation of Cav2 calcium channels. Pharmacol Rev 58:837-862.

Varela D, Niemeyer MI, Cid LP, Sepúlveda FV (2002) Effect of an $\mathrm{N}$-terminus deletion on voltage-dependent gating of the ClC-2 chloride channel. J Physiol (Lond) 544:363-372.

Wang ZW, Saifee O, Nonet ML, Salkoff L (2001) SLO-1 potassium channels control quantal content of neurotransmitter release at the C. elegans neuromuscular junction. Neuron 32:1-20.

Wei A, Jegla T, Salkoff L (1996) Eight potassium channel families revealed by the C. elegans genome project. Neuropharmacology 35:805-829.

Yuan A, Dourado M, Butler A, Blanton N, Wei A, Salkoff L (2000) SLO-2, A $\mathrm{K}^{+}$channel with a unique $\mathrm{CI}^{-}$dependence. Nat Neurosci 3:771-779. 